\title{
Microcapsules made of sodium alginate for the prolonged release of phenibut
}

\author{
Yulia POLKOVNIKOVA 1 * iD, Kseniya KORYANOVA 2 (D) \\ 1 Federal State Budget Educational Institution of Higher Education "Voronezh State University", Russia, 394036, \\ Voronezh, ul. Student, 3. \\ 2 Pyatigorsk Medical and Pharmaceutical Institute - Branch of FGBOU in VolgGMU Ministry of Health care of Russia, \\ Russia, 357500, Pyatigorsk, Kalinin Ave., 11. \\ * Corresponding Author. E-mail: juli-polk@mail.ru (Y.P.); Tel. +79-081-39 7582.
}

Received: 08 April 2019 / Revised: 05 October 2019 / Accepted: 05 October 2019

ABSTRACT: A prolonged system of the pharmaceutical substance delivery with nootropic effect was developed; it was phenibut in the form of microcapsules. The polymers used were sodium alginate as polyanion and Calcium chloride as polycation. It was realized by spraying a solution of sodium alginate in a solution of Calcium chloride, which induces the gelation. The features of the surface for the obtained microcapsules with the different ratio of nucleus/substance were determined in the work. Identification of phenibut in microcapsules was performed by IR-spectroscopy. Dependence of the microencapsulation efficiency on the concentration of film-forming material solution and the ratio of nucleus/substance was found. The process of phenibut release from microcapsules in the medium of $0.1 \mathrm{M}$ solution of hydrochloric acid was investigated.

KEYWORDS: Microencapsulation; extrusion; IR-spectroscopy; microencapsulation efficiency; release kinetics; morphology; phenibut.

\section{INTRODUCTION}

Over the past few decades, the rise of modern pharmaceutical technology and the amazing growth of the biotechnology industry have revolutionized the approach to drug discovery and development.

The most common method of administration of drugs is in the form of pills or injections. These methods of administration meet the requirements of efficacy for several drugs. However, these methods are inadequate for many new drugs. To overcome these difficulties, new technologies, like the microencapsulation, have been developed $[1,2]$.

These technologies are based on the use of polymers [3-5]. Polysaccharides, such as alginate, have been widely used in the microencapsulation technology.

Probably, the most important property of alginates is their ability to form gels by reaction with divalent cations such as calcium or barium by binding between guluronic acid blocks in alginate and the divalent cations. These gels are similar to solids because they retain their shape and resist stress. This process of gelation is an almost instantaneous and irreversible process, which is governed by the relative rate of diffusion of barium ions and polymer molecules into the gelling zone [6-8].

A lot of microencapsulation methods involve one of two heavy-load conditions (contact with organic solvent and/or thermal contact during treatment) that is usually a problem, especially during a treatment of biomaterials [9-15].

Vascular encephalopathy takes the second place in the structure of mortality as a result of circulatory system diseases [16,17]. Annual death rate from the stroke is one of the highest in the world. It should be noted an important physiological role of gamma aminobutyric acid (GABA) in the regulation of the functional activity of central nervous system for these kinds of diseases $[18,19]$.

At present, the establishment of the new drug formulations for such derivative of GABA as $\gamma$-amino- $\beta$ phenylbutyric acid hydrochloride (phenibut) characterized by a prolonged action is quite actual $[20,21]$.

The aim of the work is the development of a system for prolonged release of nootropic effect. It is based on the use of sodium alginate microcapsules charged with phenibut.

How to cite this article: Polkovnikova Y, Koryanova K. Microcapsules made of sodium alginate for the prolonged release of phenibut. J Res Pharm. 2019; 23(6): 1040-1047. 


\section{RESULTS AND DISCUSSION}

\subsection{Morphology of microcapsules}

Using extrusion technique microcapsules of sodium alginate were obtained charged with phenibut.

Porosity of the microcapsules structure is known to be likely influenced by the composition and the ratio of extracted complexes. Therefore, an influence of the ratio of nucleus/polymer on morphology of the obtained samples was studied in the work.

Rough surface with a lot of tension bars can be well observed in the micrographs of the obtained polymer microcapsules (Figures 1 and 2).
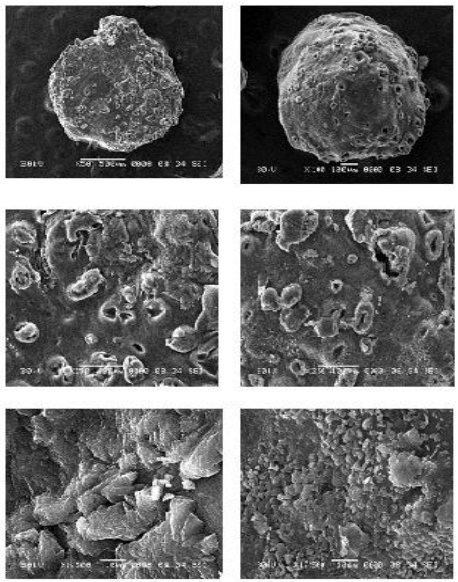

$0,5 \%$

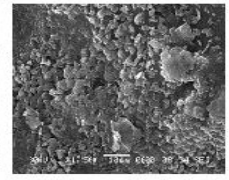

$1 \%$
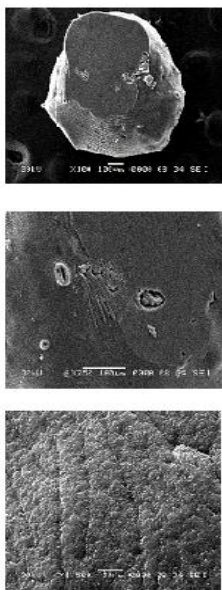

$1,5 \%$
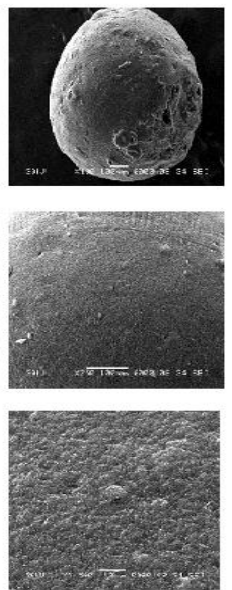

$2 \%$
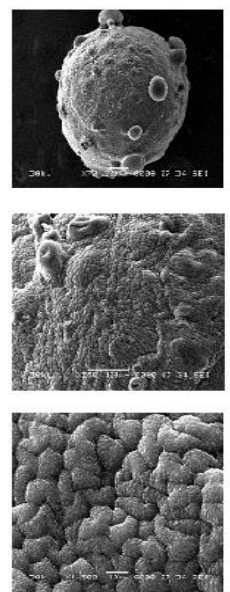

$2,5 \%$
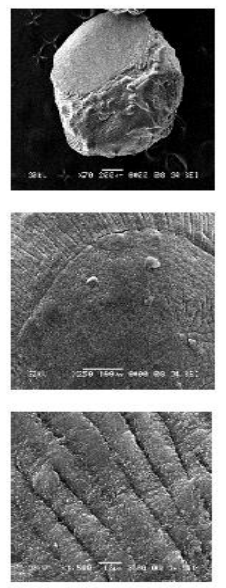

$3 \%$

Figure 1. Microphotographs of the microcapsules surface of sodium alginate with phenibut (ratio 1:1).
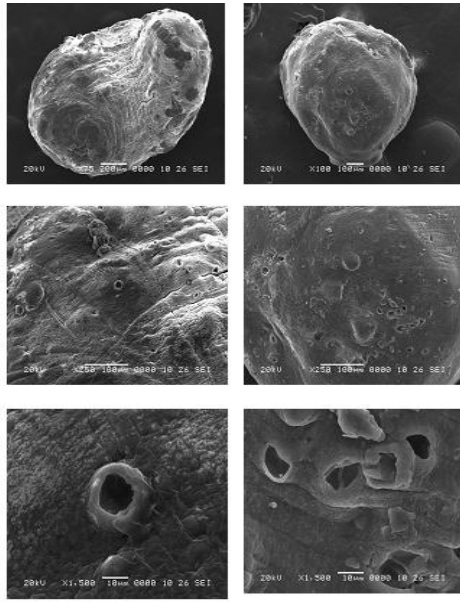

$0,5 \%$
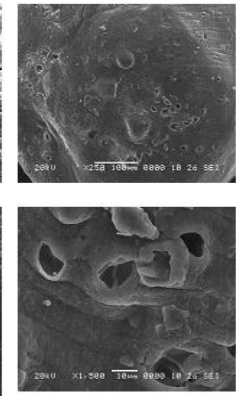

$1 \%$
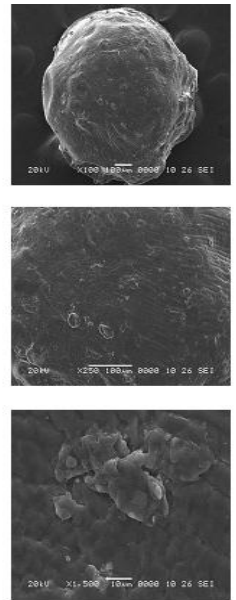

$1,5 \%$
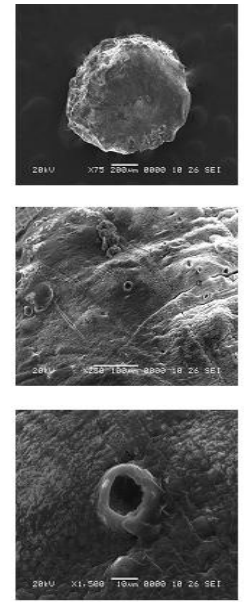

$2 \%$
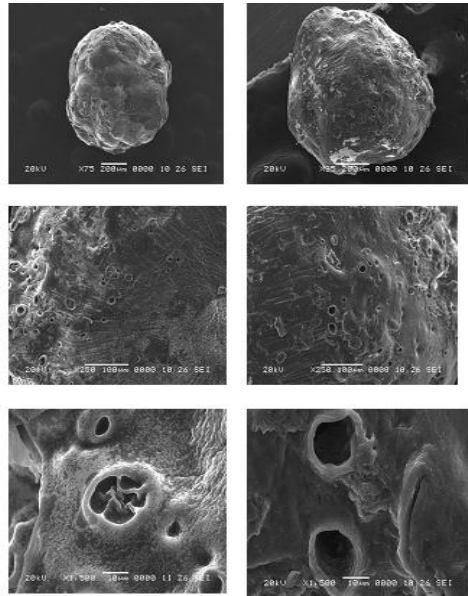

$2,5 \%$

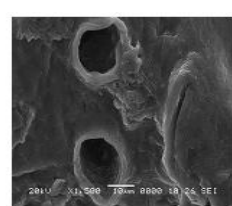

$3 \%$

Figure 2. Microphotographs of the microcapsules surface of sodium alginate with phenibut (ratio 0.5:1).

The obtained microcapsules are characterized by relief, porous surface and the presence of the bulk «halls». Surface porosity and formation of «halls» depend on the ratio of nucleus/polymer. For example, if thee ratio of nucleus/polymer is of 0,5:1 more even surface of microcapsules can be noticed with little characteristic «folds». At the same time microcapsules surface with the ratio of nucleus/polymer equal to 1:1 is presented with deep folds and tension bars.

\subsection{Phenibut identification}

Results of IR-spectroscopy study for a substance of phenibut, microcapsules-placebo and microcapsules with phenibut are presented in Figure 3. 


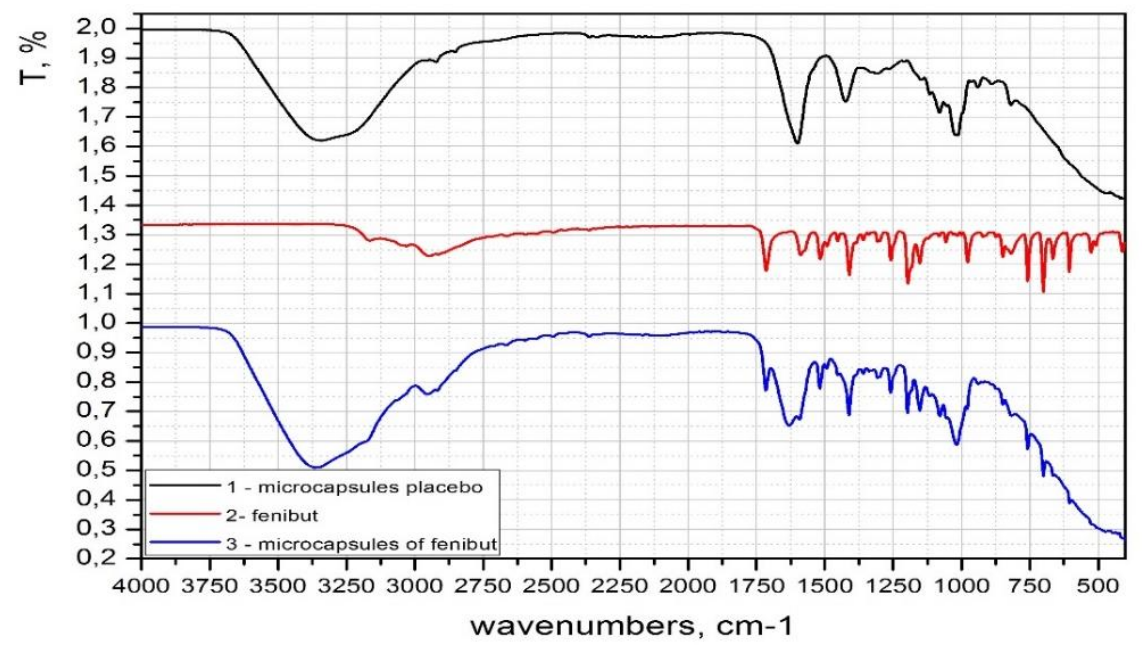

Figure 3. IR-transmission spectra of phenibut substance, microcapsules-placebo and microcapsules with phenibut.

Comparison of IR-spectra made it possible to identify phenibut substance in the microcapsues. IRspectra of the substance and microcapsules with phenibut within the range of $4000-400 \mathrm{~cm}^{-1}$ show absorption bands at 3050-2800 $\mathrm{cm}^{-1}$, meaning the presence of the primary aliphatic aminogroup in the samples; while the bands at 1712,1656, 1668, 1620 indicate at the presence of carboxylic group in the same samples and thus allowing to state that chemical interaction between the chosen components of the mixture is absent.

\subsection{Microencapsulation efficiency}

Calibration plot for the dependence of the optical density on the amount of phenibut in a solution appears as a straight line (Figure 4).

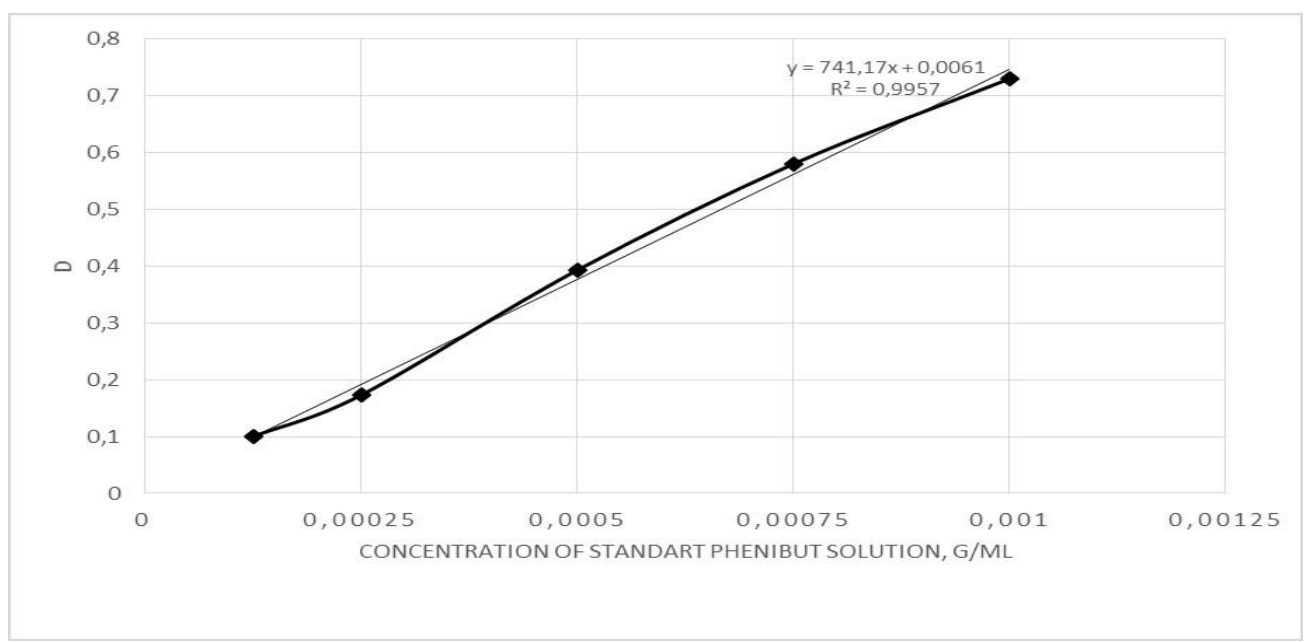

Figure 4. Calibration curve for the dependence of the optical density on phenibut concentration in a solution.

Efficiency of phenibut microencapsulation in a dependence on the concentration of film-forming material solution and the ratio of nucleus/polymer is presented in Table 1.

From Table 1 it follows that the efficiency of microencapsulation is considerably effected as by the concentration of film-forming material as by the ratio of nucleus/polymer. For example with an increase of concentration of sodium alginate solution efficiency of microencapsulation is also enhanced and attains 28.1 $\%$ at the concentration of sodium alginate solution of $2 \%$ and the ratio of nucleus/polymer 1:1. For the concentration of sodium alginate solution more than $2 \%$ a considerable decrease of efficiency is noticeable as for the ratio of nucleus/polymer 1:1, as for that one of 0.5:1. 
Table 1. Efficiency of phenibut microcapsulation.

\begin{tabular}{ccc}
\hline $\begin{array}{c}\text { Concentration of } \\
\text { sodium alginate, } \%\end{array}$ & \multicolumn{2}{c}{ Microcapsulation efficiency, $\%$} \\
\cline { 2 - 3 } & $\mathbf{0 . 5 : 1}$ & $\mathbf{1 : 1}$ \\
\hline 0.5 & 17.5 & 12.0 \\
1.0 & 23.1 & 12.5 \\
1.5 & 26.9 & 25.2 \\
2.0 & 18.8 & 28.1 \\
2.5 & 22.5 & 15.6 \\
3.0 & 21.3 & 10.1 \\
\hline
\end{tabular}

\subsection{Examination of phenibut release from polymer microstructures in vitro}

In order to make a comparative estimation of a release degree of the reactant from pharmaceutical substances investigations of the release degree of phenibut into the medium of $0.1 \mathrm{M}$ solution of hydrochloric acid were performed.

Figures 5 and 6 represent the profiles (or curves) of release of the pharmaceutical substance into dissolution medium (\%) in a dependence on time (min) (RSD, \%).

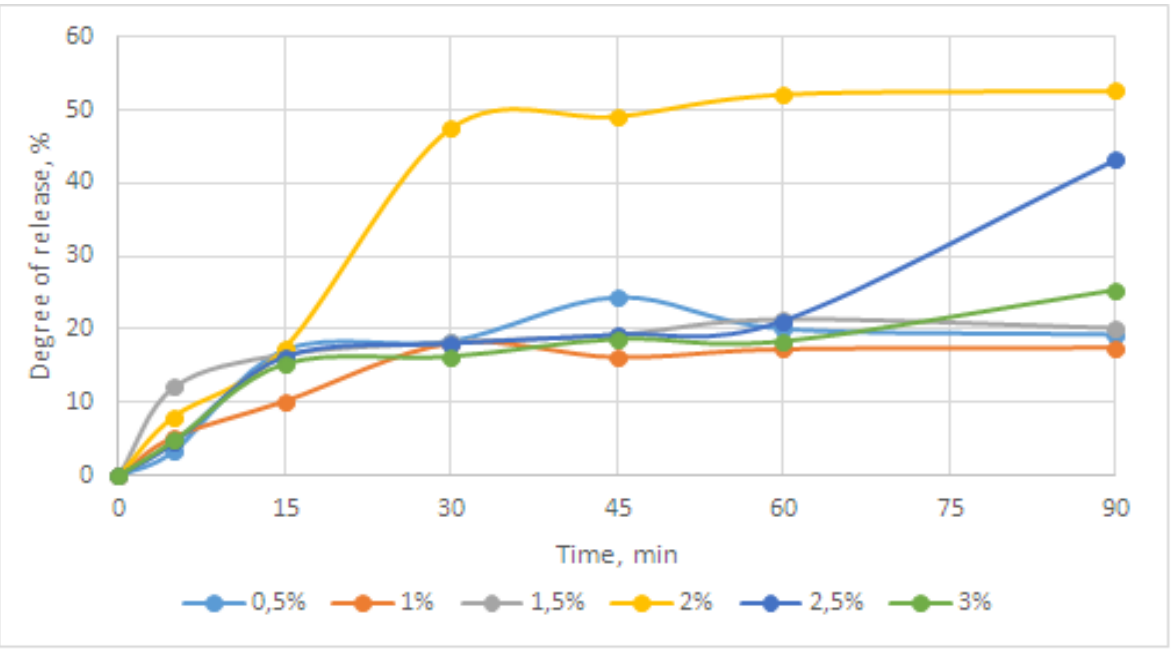

Figure 5. Dynamics of phenibut release from microcapsules $(0,5: 1)$.

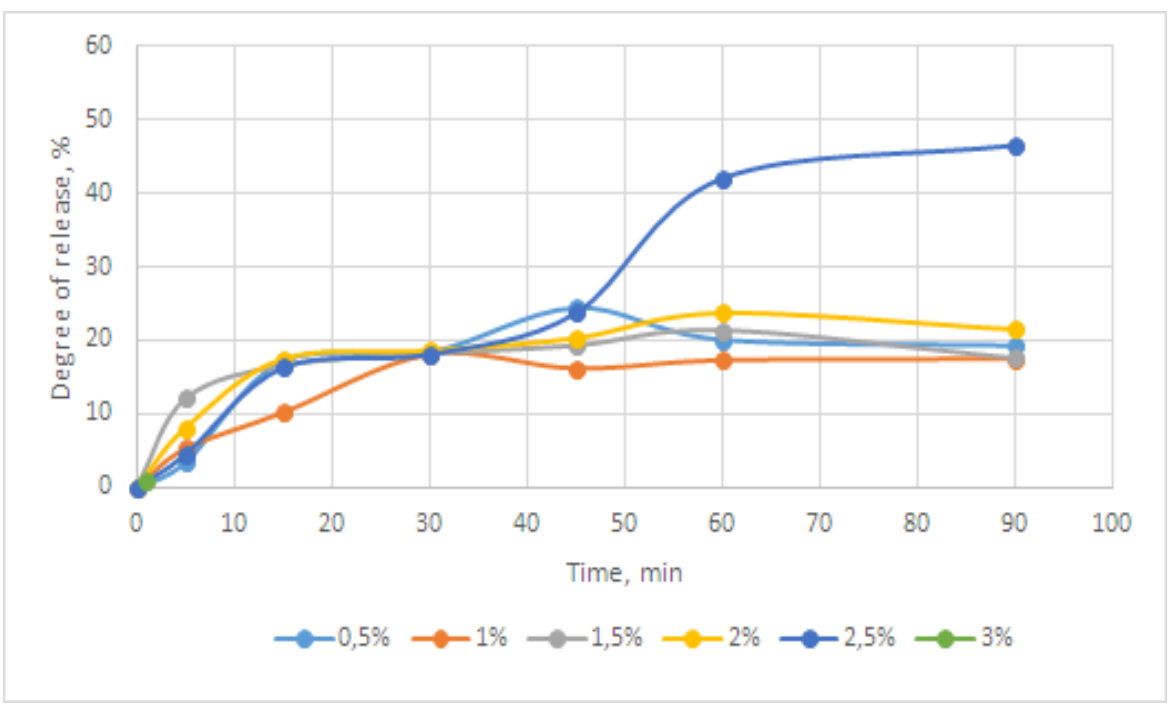

Figure 6. Dynamics of phenibut release from microcapsules (1:1). 
As a result of the performed biopharmaceutical investigations it was shown the influence of concentration of the film-forming material solution and the ratio of nucleus/polymer on a release of phenibut into dissolution medium.

\subsubsection{Ratio of nucleus/polymer 1:1}

The observed homogeneous release of the pharmaceutical substance takes place from microcapsules for all the concentrations of the film-forming material solution. Most complete release of phenibut is observed from the microcapsules with the concentration of the film-forming material solution of $2 \%$; at 90 min of the experiment concentration of the substance is of $52.2 \%$. Slightly less release of phenibut was observed for the microcapsules with the concentration of the film-forming material solution of 0.5 and $1 \%$ and it was of 19.3 and $17.5 \%$, respectively (Figure 5 ).

RSD, \% is presented in Table 2.

Table 2. RSD, \% (Ratio of nucleus/polymer 1:1).

\begin{tabular}{cccccc}
\hline $\begin{array}{c}\text { Concentration of sodium } \\
\text { alginate, } \mathbf{0}\end{array}$ & $\mathbf{5}$ & $\mathbf{3 0}$ & $\mathbf{4 5}$ & $\mathbf{6 0}$ & $\mathbf{9 0}$ \\
\cline { 2 - 6 } & 3.8 & 2.5 & 2.4 & 2.5 & 4.8 \\
1.0 & 1.8 & 0.7 & 1.5 & 1.6 & 4.9 \\
1.5 & 2.9 & 4.9 & 2.0 & 4.3 & 2.6 \\
2.0 & 4.6 & 5.0 & 3.9 & 3.7 & 3.5 \\
2.5 & 2.8 & 3.1 & 4.7 & 4.3 & 4.6 \\
3.0 & 2.0 & 1.9 & 4.0 & 3.0 & 4.1 \\
\hline
\end{tabular}

\subsubsection{Ratio of nucleus/polymer 0.5:1}

The observed homogeneous release of the pharmaceutical substance takes place from microcapsules for all the concentrations of the film-forming material solution. Most complete release of phenibut is observed from the microcapsules with the concentration of the film-forming material solution of $2.5 \%$; at 90 min of the experiment concentration of the substance is of $46.8 \%$. Slightly less release of phenibut was observed for the microcapsules with the concentration of the film-forming material solution of 1 and $1.5 \%$ and it was of 17.7 and $18.3 \%$, respectively (Figure 6). RSD, \% is presented in Table 3.

Table 3. RSD, \% (Ratio of nucleus/polymer 0,5:1).

\begin{tabular}{cccccc}
\hline $\begin{array}{c}\text { Concentration of sodium } \\
\text { alginate, } \mathbf{0}\end{array}$ & $\mathbf{1 5}$ & $\mathbf{3 0}$ & $\mathbf{4 5}$ & $\mathbf{6 0}$ & $\mathbf{9 0}$ \\
\cline { 2 - 6 } & 15 & 1.9 & 4.4 & 4.2 & 4.2 \\
1.5 & 3.1 & 3.8 & 4.5 & 3.8 & 4.0 \\
1.5 & 5.0 & 5.0 & 3.9 & 4.7 & 2.9 \\
2.0 & 3.2 & 4.8 & 4.3 & 4.9 & 2.7 \\
2.5 & 3.8 & 4.2 & 3.7 & 5.0 & 3.6 \\
3.0 & 4.4 & 3.9 & 4.5 & 3.8 & 3.9 \\
\hline
\end{tabular}

Thus, in the study it was determined that a release proceeds more completely and homogeneously from microcapsules with the ratio of nucleus/polymer 1:1 (concentration of sodium alginate solution of $2 \%$ ) and it attains $52.2 \%$ after 90 minutes of experiment.

\section{CONCLUSIONS}

As a result of the research, modeling samples of phenibut microcapsules were obtained. It was stated, that with the increasing concentration of sodium alginate solution, the efficiency of microcapsulation increases, and reaches $28.1 \%$ at a concentration of $2 \%$ sodium alginate solution (microcapsules with chitosan). In the study of the influence of the concentration of sodium alginate solution on the degree of release of Phenibut from microcapsule samples, it was found that at a concentration of $1 \%$, the degree of release is 17.5 $\%$ (core/polymer ratio $1: 1$ ) and $17.7 \%$ (core/ polymer ratio $0.5: 1$ ). The results which were obtained in the study 
are promising for further detailed study of microcapsulated phenibut in order to create its pharmaceutical form.

\section{MATERIALS AND METHODS}

Substance of $\gamma$-amino- $\beta$-phenylbutyric acid hydrochloride (Figure 7 ), corresponding to the requirements ND 42-14132-06, sodium alginate FOODALGA of 500 grade, «NevaReaktiv».

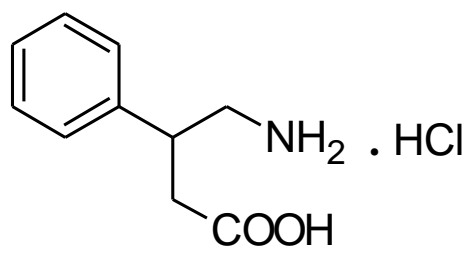

Figure 7. Structural formula of phenibut hydrochloride.

\subsection{Obtaining of microcapsules with a shell of sodium alginate}

At the first stage the process of cross-linking of alginic acid with calcium ions was examined.

Phenibut immobilization by incorporation was performed according to the following technique: a sample of $1.0 \mathrm{~g}$ of phenibut was placed into solution of sodium alginate (concentration of $0.5 ; 1 ; 1.5 ; 2 ; 2.5 ; 3 \%$ ) and dosed with the use of extrusion through a sprayer into $0.2 \mathrm{M}$ solution of calcium chloride. The ratio of volume of the film-forming material solution : calcium chloride solution 1:10. The formed particles of calcium alginate were sustained in a cross-linking agent solution for 20 minutes for the hardening.

Alginic acid is a block-copolymer, formed by polymers of three types: blocks of homopolymers of Dmannuronic and L-guluronic acid and mixed heteropolymeric blocks containing both types of monomeric chains. Residues of uronic acids in alginic acid are bound with $\beta-1,4$-glycosidic linkages in the form of linear chain.

When formation of microcapsules a reaction of substitution of $\mathrm{Na}+$ cations with $\mathrm{Ca}^{2+}$ cations proceeds with the formation of calcium alginate insoluble in water.

Structure of the surface provides an important information on porosity of microsystems intended for a delivery of the pharmaceutical substances. Scanning electron microscopy (SEM) was applied for the study of morphological features of the obtained samples and it was realized with the use of microscope (JSM-6380LV, JEOL).

Gold was deposited on the samples while performing of morphological investigations with the use of SEM in order to reduce the charging effects.

\subsection{Identification of phenibut}

IR-spectra were surveyed with Vertex 70 spectrometer (Bruker Optik GmbH, Germany), in the middle part of IR-region in the range of $4000-400 \mathrm{~cm}^{-1}$ applying ATR technique (attenuated total reflectance method), using ZnSe attachment with the diamond window; as a result, IR-absorption spectra were obtained for phenibut substance, placebo-microcapsules and microcapsules with phenibut.

\subsection{Efficiency of microencapsulation}

A key point in obtaining of the microencapsulated product is the efficiency of micro- capsulation, i.e. a part of the encapsulated substance involved inside the capsules related to its reference quantity. Efficiency of micro-capsulation was determined in a «direct» way.

Weighed microcapsules of $50 \mathrm{mg}$ mass were dissolved in $10 \mathrm{ml}$ of $0.1 \mathrm{M}$ of hydrochloric acid solution for 30 minutes under agitation with magnetic stirrer in a closed vessel. Optical density of the obtained solution was determined by spectrophotometry technique with spectrophotometer Hitachi U-1900 at the wavelength of $257 \pm 2 \mathrm{~nm}$. Solution of $0.1 \mathrm{M}$ hydrochloric acid was used as a reference one. Concentration of a substance in solution was determined with the use of calibration plot.

To obtain calibration plot for the dependence of the optical density on the concentration of the standard sample of phenibut a precise sampling of $0.25 \mathrm{~g}$ phenibut was transferred to the volumetric flask of $250 \mathrm{ml}$ capacity, then added $200 \mathrm{ml}$ of hydrochloric solution and finally brought the volume of solution up to the required label. 
To prepare dilutions aliquots of the reference standard were transferred into the volumetric flasks of 25 $\mathrm{ml}$ in volume and brought the volume of solution up to the label with $0.1 \mathrm{M}$ of hydrochloric acid solution. Aliquots of standard solution were used, in ml: $0.5 ; 1.0 ; 1.5 ; 2.0 ; 2.5 ; 3.0 ; 3.5$.

After measuring of the amount of phenibut released under dissolution and knowing its initial concentration it was possible to calculate efficiency of microencapsulation taking into account the amount of substance involved in microcapsules Ccaps., as compared with the amount of the initially dissolved substance Cinit. According to the formula (Eq. 1):

$$
\mathrm{E}=\frac{m_{\text {caps }}}{m_{\text {inform }}} * 100 \%
$$

Eq. 1

\subsection{Examination of phenibut release from microstructures in vitro}

«Dissolution» test was performed with the help of dissolution tester DT 626/1000HH produced by ERWEKA Company (Germany) provided with impeller mixer.

Dissolution medium was as follows: 0,1 M solution of hydrochloric acid, the volume of dissolution medium was of $700 \mathrm{ml}$, time points for sampling were as follows: $15 \mathrm{~min}, 30 \mathrm{~min}, 45 \mathrm{~min}, 60 \mathrm{~min}, 90 \mathrm{~min}, 120$ $\min , 180 \mathrm{~min}$.

1 capsule with the examined microcapsules was placed in each of 6 glasses. The glasses were immersed in the vessels for dissolution with $700 \mathrm{ml}$ of dissolution medium $(0.1 \mathrm{M}$ solution of hydrochloric acid), preliminarily temperature-controlled at $37 \pm 0.5{ }^{\circ} \mathrm{C}$. After the above indicated periods of time $5 \mathrm{ml}$ of the medium was sampled. After sampling the specimens were filtered through the membrane filters with a diameter of pores $0,45 \mu \mathrm{m}$ and the first portions of filtrate were removed.

$0.1 \mathrm{M}$ solution of hydrochloric acid was used as a reference solution. Concentration of substance in solution was determined with the help of calibration plot.

Statistical treatment of the experimental results was performed with the use of Microsoft Office Excel 2013 suite calculating the average amount of dissolved substance and the relative standard deviation (RSD,\%).

Authorship statement: Author contributions: Concept -Y.P.; Design -Y.P.; Supervision -Y.P., K.K.; Resource Y.P., K.K.; Materials -Y.P., K.K. Data Collection and/or Processing -Y.P.; Analysis and/or Interpretation -Y.P.; Literature Search -Y.P., K.K.; Writing -Y.P., K.K.; Critical Reviews -Y.P., K.K.

Conflict of interest statement: The authors declared no conflict of interest.

\section{REFERENCES}

[1] Hojjati M, Razavi SH, Rezaei K. Gilani K. Spray drying microencapsulation of natural canthaxantin using soluble soybean polysaccharide as a carrier. Food Sci Biotechnol. 2011; 20: 63-69. [CrossRef]

[2] Fuensanta M, Grau A, Romero-Sánchez MD, Guillem C, López-Buendía MÁ. Effect of the polymer shell in imidazole microencapsulation by solvent evaporation method. Polym. Bull. 2013; 70: 3055-3074. [CrossRef]

[3] Mano JF. Stimuli-responsive polymeric systems for biomedical applications. Adv Eng Mater. 2008; 10: 515-527. [CrossRef]

[4] Goh CH, Heng PWS, Chan LW. Alginates as a useful natural polymer for microencapsulation and therapeutic applications. Carbohydr Polym. 2012; 88: 1-12. [CrossRef]

[5] Xie J, Wang CH Electrospray in the dipping mode for cell microencapsulation. J Colloid Interface Sci. 2007; 312: 247255. [CrossRef]

[6] Xu J, Li S, Tan J, Luo G. Controllable preparation of monodispersed calcium alginate microbeads in a novel microfluidic system. Chem Eng Technol. 2008; 31: 1223-1226. [CrossRef]

[7] Zhong D, Huang X, Yang H, Cheng R. New insights into viscosity abnormality of sodium alginate aqueous solution. Carbohydr Polym. 2011; 81(4): 948-9. [CrossRef]

[8] Chan ES, Wong SL, Lee PP, Lee JS, Ti TB, Zhang Z, Poncelet D, Ravindra P, Phan SH, Yim ZH. Effects of starch filler on the physical properties of lyophilized calcium-alginate beads and the viability of encapsulated cells. Carbohydr Polym. 2011; 83(1): 225-232. [CrossRef]

[9] Agnihotri SA, Aminabhavi TM. Controlled release of clozapine through chitosan microparticles prepared by a novel method. Journal of Controlled Release. 2004; 96(2): 245-259. [CrossRef] 
[10] Li J, Zhang JJ, Zhao XJ. Preparation of porcine hemoglobin microcapsules of chitosan-sodium alginate. Frontiers of Chemistry in China. 2007; 2(3): 315-317. [CrossRef]

[11] El-Gibaly I. Development and in vitro evaluation of novel floating chitosan microcapsules for oral use: comparison with non-floating chitosan microspheres. Int J Pharm. 2002, 249(1-2): 7-21. [CrossRef]

[12] Lin WC, Yu DG, Yang MC. pH-Sensitive polyelectrolyte complex gel microspheres composed of chitosan/sodium tripolyphosphate/dextran sulfate: swelling kinetics and drug delivery properties. Colloid Surface B: Biointerfaces. 2005; 44(2-3): 143-151. [CrossRef]

[13] Wang CY, Ye SQ, Dai L, Liu XX, Tong Z. Enhanced resistance of polyelectrolyte multilayer microcapsules to pepsin erosion and release properties of encapsulated indomethacin. Biomacromolecules. 2007; 8: 1739-1744. [CrossRef]

[14] Anal AK, Stevens WF, Remunan-lopez C. Ionotropic cross-linked chitosan microspheres for controlled release of ampicillin. Int J Pharm. 2006; 312(1-2): 166-173. [CrossRef]

[15] Om Prakash Ranjan, Gopal Venkatesh Shavi, Usha Yogendra Nayak, Karthik Arumugam, Ranjith Kumar Averineni, Sreenivasa Reddy Meka, Pandey Sureshwar. Controlled release chitosan microspheres of mirtazapine: In vitro and in vivo evaluation. Arch Pharm Res. 2011; 34(11): 1919-1929. [CrossRef]

[16] Giordano C, Viscomi C, Orlandi M, Paola PapoffAlberto SpaliceAlberto BurlinaIvano Di MeoValeria TirantiVincenzo LeuzziGiulia d'Amati Massimo Zeviani. Morphologic evidence of diffuse vascular damage in human and in the experimental model of ethylmalonic encephalopathy. J Inherit Metab Dis. 2012; 35: 451-458. [CrossRef]

[17] Crona DJ, Whang YE. Posterior reversible encephalopathy syndrome induced by enzalutamide in a patient with castration-resistant prostate cancer. Invest New Drugs. 2015; 33: 751-754. [CrossRef]

[18] Rivera-Ingraham GA., Espinosa F, Krock B. Presence of Gamma-Aminobutyric Acid (Gaba) in the Pedal Mucus of the Critically Endangered Species Patella ferrugineaJ Chem Ecol. 2015; 41: 501-504. [CrossRef]

[19] Yu HH, Choi JH, Kang KM. Potential of a lactic acid bacterial starter culture with gamma-aminobutyric acid (GABA) activity for production of fermented sausage. Food Sci Biotechnol. 2017; 26: 1333-1341. [CrossRef]

[20] Polkovnikova YA, Glushko AA, Mikhailovskaya IY, Karieva YS. Simulation of vinpocetine release process from microcapsules with hydrophobic shell. Pharmacy \& Pharmacology. 2017; 5(4): 344-367. [CrossRef]

[21] Zavadenko NN, Suvorinova NY. Results of Pharmacotherapy of Attention Deficit Hyperactivity Disorder: Assessment Using Neuropsychological Methods. Neurosci Behav Physi. 2016; 46: 354-359. [CrossRef]

This is an open access article which is publicly available on our journal's website under Institutional Repository at http://dspace.marmara.edu.tr. 